\title{
Genetic signature and treatment of pediatric high-grade glioma
}

\author{
MILENA GUIDI $^{1}$, LAURA GIUNTI $^{2}$, ANNA MARIA BUCCOLIERO $^{3}$, CHIARA CAPORALINI $^{3}$, \\ MARIA LUIGIA CENSULLO ${ }^{1}$, LUISA GALLI ${ }^{4}$, LORENZO GENITORI ${ }^{5}$ and IACOPO SARDI ${ }^{1}$ \\ ${ }^{1}$ Neuro-Oncology Unit, Department of Pediatric Oncology, ${ }^{2}$ Medical Genetics Unit, ${ }^{3}$ Pathology Unit, \\ Meyer Children's University Hospital; ${ }^{4}$ Department of Health Sciences, University of Florence, \\ ${ }^{5}$ Neurosurgery Unit, Meyer Children's University Hospital, I-50139 Florence, Italy
}

Received January 7, 2020; Accepted November 13, 2020

DOI: $10.3892 /$ mco.2021.2232

\begin{abstract}
Pediatric high-grade glioma (HGG) is a type of malignancy that carries a poor prognosis. The genetic analysis of HGGs has previously identified useful mutations, the targeting of which has improved prognosis. Thus, further research into the more common mutations, such as $\mathrm{H} 3$ histone variants (HIST1H3B and H3F3A) and BRAF V600E, may be useful in identifying tumors with different prognoses, as the mutations are considered to drive two distinct oncogenic programs. The present study performed a retrospective analysis of pediatric HGGs. In total, 42 cases of HGG, including 32 cases $(76.1 \%)$ of anaplastic astrocytoma and 10 cases (23.8\%) of glioblastoma multiforme (GBM), were assessed. The median age of the patients was 7 years (range, 0-32 years). Mutations on histone H3, in particular the K27M and G34R mutations in the distinct variants HIST1H3B and H3F3A, in addition to the presence of the BRAF V600E mutation, were analyzed in 24 patients. The H3F3A K27M mutation was identified in 7 patients (29.1\%), while the HIST1H3B K27M mutation was only observed in 1 patient with GBM. In addition, 5 patients harbored a BRAF V600E mutation (21\%), while the H3F3A G34R mutation was not recorded in any of the patients. The overall survival of the wild-type patients at 20 months was $68 \%$ [confidence interval (CI): $38-85 \%$ ] compared with $28 \%$ (CI: $0.4-60 \%$ ) in patients with the H3F3A $\mathrm{K} 27 \mathrm{M}$ mutation. These results suggested that patients with
\end{abstract}

Correspondence to: Dr Iacopo Sardi, Neuro-Oncology Unit, Department of Pediatric Oncology, Meyer Children's University Hospital, 24 Viale Gaetano Pieraccini, I-50139 Florence, Italy

E-mail: iacopo.sardi@meyer.it

Abbreviations: AA, anaplastic astrocytoma; CNS, central nervous system; DIPG, diffuse intrinsic pontine glioma; GBM, glioblastoma multiforme; HDCT, high-dose chemotherapy; HGG, high-grade glioma; LGG, low-grade glioma; OS, overall survival; PFS, progression-free survival; SDCT, standard-dose chemotherapy

Key words: glioblastoma, high-grade glioma, histone 3, midline glioma, oncology, pediatric, H3F3A K27M, BRAF V600E the H3F3A K27M mutation had a worse prognosis compared with wild-type patients $(\mathrm{P}=0.0045)$. Moreover, $3 / 5$ patients with the BRAF V600E mutation had HGGs that were derived from a previous low-grade glioma ( $\mathrm{LGG} ; \mathrm{P}=0.001$ ). In conclusion, these results suggested that histone $\mathrm{H} 3$ mutations may help predict the outcome in patients with HGG. In addition, the BRAF V600E mutation was found to be associated with an increased risk of anaplastic progression. The novel data of the present study may help better define the clinical and radiological characteristics of glioma.

\section{Introduction}

Anaplastic astrocytoma (AA) and glioblastoma (GBM) represent $\sim 20 \%$ of all central nervous system (CNS) tumors. These two brain tumor subtypes respond poorly to therapy and they are the most biologically aggressive malignancies and the principal cause of cancer-related mortality and morbidity during childhood (1). Although the outcome of pediatric high-grade gliomas (HGGs) is marginally superior to that of their adult counterparts, it remains poor, despite aggressive treatment (2).

The two main prognostic factors are the primary location and resectability. Currently, up to $85 \%$ of patients with hemispheric GBM succumb to the disease within 2 years of diagnosis (3). The genetic alterations involved in the pathogenesis of pediatric HGGs, particularly the role of somatic driver mutations (K27M) in the histone $\mathrm{H} 3$ genes (H3F3 and H1ST1H3B), were previously investigated. Another mutation in $\mathrm{H} 3 \mathrm{~F} 3 \mathrm{~A}, \mathrm{G} 34 \mathrm{R} / \mathrm{V}$, has also been described, and appears to be more frequent among young adults (4).

The histone methyltransferases are responsible for the methylation of histone $\mathrm{H} 3$ at Lys27 and the K27M mutation replaces lysine with methionine, while the G34V/R mutation in amino acid 34 initiates the replacement of glycine with either valine or arginine (5). Histone proteins undergo modifications, such as acetylation, methylation and phosphorylation, which are mediated by writers (methyltransferases), erasers (demethylases) and readers (which recognize specific histone modifications). These mechanisms on histone tails are important in facilitating gene transcription (6). It is well-established that the misregulation of histone lysine methylation is implicated in the occurrence and development of numerous types of cancer (4); however, the role of the K27M mutation in the development of glioma remains unclear. 
Considering the clinical implications, H3.3K27M mutations have been found to be associated with poor prognosis of pediatric HGGs, which appear to have diffuse growth patterns and a midline localization. This group is classified as diffuse midline glioma, H3K27M-mutant, and includes previously defined entities, such as diffuse intrinsic pontine glioma (DIPG) (7).

Furthermore, the two main histone H3 variants have been associated with distinct biological characteristics, thereby driving different oncogenic programs. Tumors with mutated H1ST1H3B were found to be less aggressive and more responsive to radiotherapy and standard treatment. Histone H3F3A and HIST1H3B K27M mutations in HGGs help define the two subgroups of HGG, which exhibit different prognoses and phenotypes (8).

The BRAF V600E mutation is commonly present in xanthoastrocytomas and gangliogliomas, but it has also been described in diffuse astrocytoma and low-grade glioma (LGG). It was previously demonstrated that the presence of mutations has diagnostic, prognostic and therapeutic potential. In fact, BRAF mutations may help differentiate benign from aggressive cancers; tumors with a BRAF V600E mutation were strongly associated with an increased risk of progression and development of a secondary cancer with anaplastic evolution. Moreover, BRAF mutations were suggested as possible targets for the improved adjuvant treatment of residual or recurrent tumors (9); no patients with double-mutant tumors (H3 and BRAF) were identified.

In the present study, the presence of mutations and their associations with clinical characteristics were compared in pediatric HGGs, with the aim to define the prognostic role of these mutations by comparing the genetic and clinical data with those reported in the existing literature.

\section{Materials and methods}

Patient studies. A total of 42 patients with HGGs recruited between January 2003 and January 2016 at the Meyer Children's University Hospital (Florence, Italy) were considered to be eligible for inclusion in the present study. Histological diagnosis and tumor grading were performed based on the 2016 World Health Organization criteria (10). The diagnoses were confirmed following review by the CNS national panel of pathologists. The study protocol was approved by the institutional ethics board and informed consent was obtained from the parents or legal guardians of all the patients.

Genetic analysis. The majority of the analyses were performed following DNA extraction from fresh-frozen tissues using a specific protocol (Qiagen $\mathrm{GmbH}$ ). The sequence analysis of the coding region of the gene was prepared using the BigDye Terminator v1.1 Cycle Sequencing kit (Applied Biosystems; Thermo Fisher Scientific, Inc.), according to the manufacturer's protocol, and sequenced on a 310 Genetic analyzer (Applied Biosystems; Thermo Fisher Scientific, Inc.). The following primer sequences were used: BRAF-EX15 forward, TCTAAGAGGAAAGATGAAGTACTATG and reverse, AGACCTTCAATGACTTTCTAGTAA; and H3F3A forward, ATCGTGGCAGGAAAAGTT and reverse, TTT AAGCAGTAGTTAAGTGTTCAAATG; and HIST1H3B
Table I. Clinical characteristics of the patients.

\begin{tabular}{lc}
\hline Characteristics & No. $(\%)$ \\
\hline Age, years & \\
Median (range) & $7(0-32)$ \\
$<3$ & 10 \\
$>3$ & 32 \\
Sex & \\
Female & $20(47.6)$ \\
Male & $22(52.4)$ \\
Tumor histology & \\
Anaplastic astrocytoma & $32(76.1)$ \\
Glioblastoma multiforme & $10(23.8)$ \\
Mutations in patients aged $>3$ years $(\mathrm{n}=24)$ & \\
H3F3A K27M & $7(29.1)$ \\
H1ST1H3B K27M & $1(4.1)$ \\
H3F3A G34R & 0 \\
BRAF V600 E & $5(20.8)$ \\
IDH & 0 \\
P53 & 0 \\
\end{tabular}

forward, GTCTCTGCAGGCAAGCTTTT and reverse, CAACTCGGTCGACTTTTGGT.

For the molecular analysis of the genes, the coding portions and the respective flanking regions were amplified by PCR. Once the systems were purified by capillary electrophoresis $(\mathrm{CE})$, software analysis was performed.

Statistical analysis. A Student's t-test was used to compare the levels between the mutated and wild-type tumors. The log-rank (Mantel-Cox) test was used to determine the association between the mutations and overall survival (OS). The patient outcome (OS) was analyzed using the following clinicopathological and treatment-related variables: Age, sex, tumor size, stage at presentation, histological subtype and response to chemotherapy. Survival was measured from the time of diagnosis to the date of death or the date of the last follow-up. Survival curves were drawn using the Kaplan-Meier product limit method. $\mathrm{P}<0.05$ was considered to indicate a statistically significant difference.

\section{Results}

Patients and treatment. A total of 42 patients were enrolled in the present study and their main clinicopathological characteristics are summarized in Table I. The median age at the time of diagnosis was 7 years (range, $0-32$ years), while 10 patients were aged $<3$ years. All subjects were treated with chemotherapy and/or radiotherapy according to the clinical studies of Meyer Children's University Hospital. The tumors comprised 10 GBMs (23.8\%) and 32 AAs (76.1\%). In total, 21 patients underwent partial resection of the lesion (R2), 11 underwent complete resection (R0) and 10 patients had a biopsy. The second observation was performed at the time of recurrence in 10 patients (1 R0, $9 \mathrm{R} 2$ ).

The 32 patients aged $>3$ years were treated with chemotherapy and radiotherapy. Patients aged $<3$ years were 


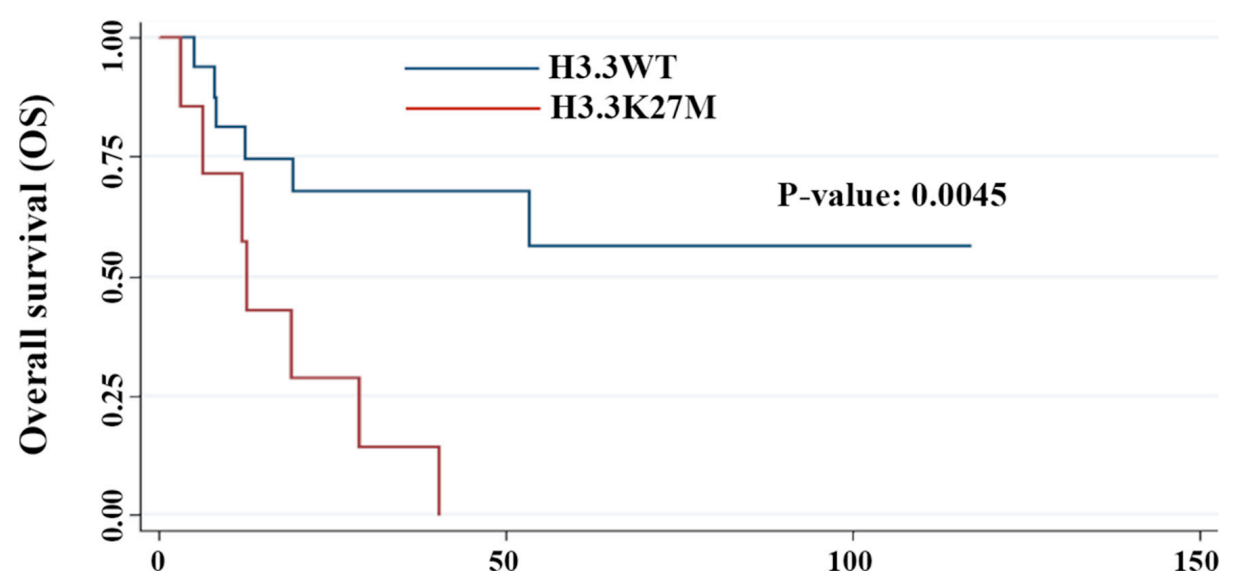

Figure 1. OS of patients with the H3.3K27M mutation and comparison with H3.3WT ( $\mathrm{P}=0.0045)$. OS, overall survival; WT, wild-type.
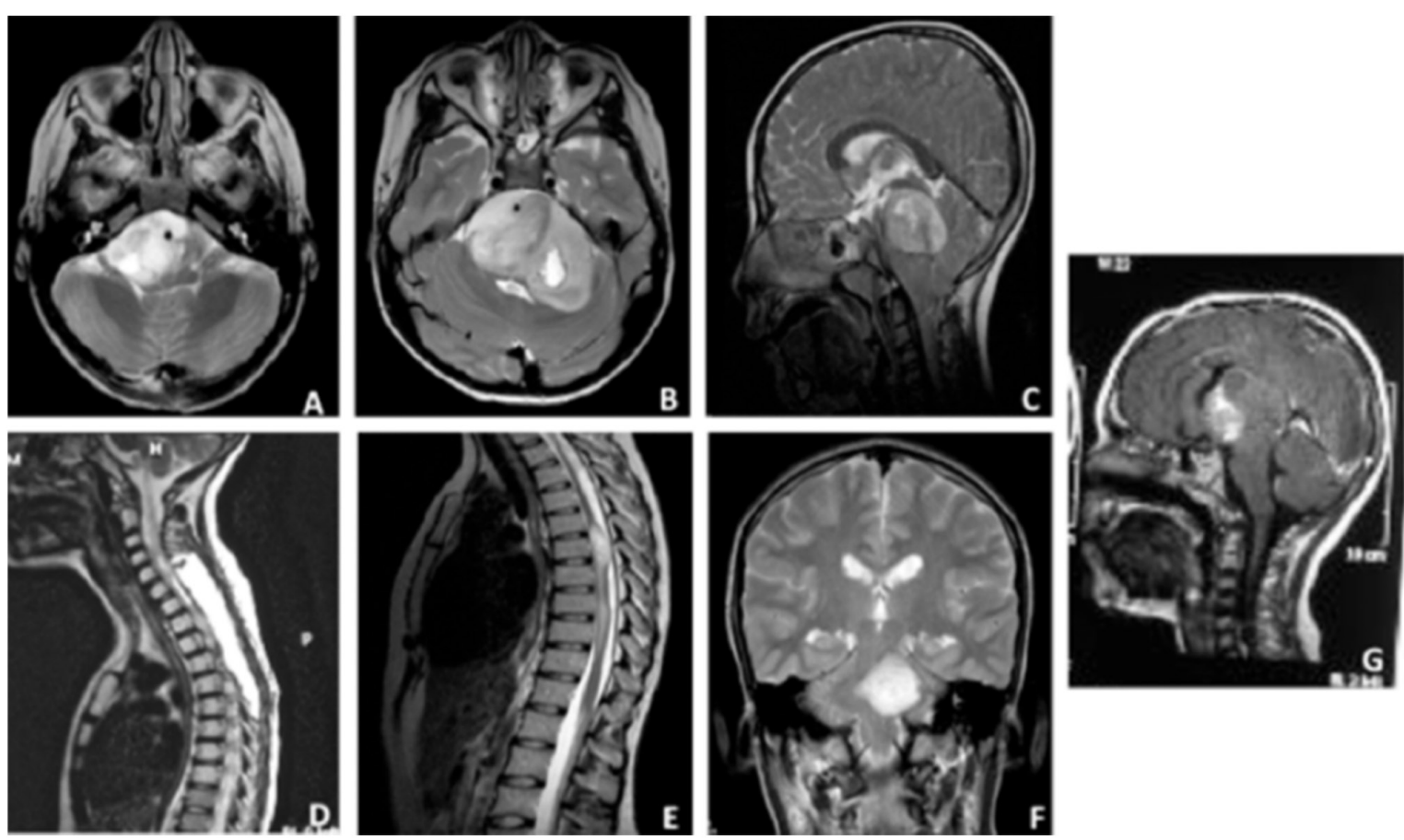

Figure 2. NMR examination of patients with H3.3K27M revealed a midline localization of the tumors. (A) Transverse NMR scan of a cerebellopontine angle tumor. (B) Transverse MRI scan of the brainstem. (C) Sagittal NMR scan of a brainstem tumor. (D and E) Sagittal NMR scans of two spinal cord tumors. (F) Coronal NMR scan of brainstem tumor. (G) Sagittal NMR scan of a pineal gland tumor. NMR, nuclear magnetic resonance.

treated with adjuvant chemotherapy [standard-dose chemotherapy (SDCT) and high-dose chemotherapy (HDCT) with autologous stem cell rescue]. Second-line chemotherapy at the time of recurrence was administered in 23 patients, 10 of whom received two lines of palliative chemotherapy.

Mutation analysis. K27M mutation analysis was performed in 24 of the patients aged $>3$ years; the mutation in histone H3.3 leading to the $\mathrm{K} 27 \mathrm{M}$ amino acid substitution was identified in $7 / 24(21 \%)$ HGGs. However, the H1ST1H3B mutation was only recorded in 1 patient with GBM. The subsequent analysis to determine the association between OS and mutations was performed using the group of 24 patients.

The H3.3K27M mutation was present in $3 \mathrm{GBM}$ and $3 \mathrm{AA}$ cases. No correlation was identified between the mutation and tumor histology $(\mathrm{P}=0.61)$. Patients with the mutation appeared to have progressive disease, but without a statistically significant correlation $(\mathrm{P}=0.07)$. However, patients carrying this mutation had a significantly worse prognosis compared with the wild-type patients ( $\mathrm{P}=0.0045$; Fig. 1). As regards localization, all the tumors with the $\mathrm{H} 3.3 \mathrm{~K} 27 \mathrm{M}$ mutation were midline tumors ( 2 in the spinal cord, 3 in the brainstem, 1 in the cerebellopontine angle and 1 in the pineal gland; Fig. 2).

The BRAF V600E mutation was identified in 5 patients (21\%), in 3 of whom the HGG developed from a previous LGG $(\mathrm{P}=0.001)$. The median age of the patients with this mutation was 6.8 years. In addition, 3 of these tumors were reported in females.

Treatment and survival. The median OS the 42 patients was 77 months. The median OS for patients aged $>3$ years was 38.5 months, while for children aged $<3$ years the median OS 


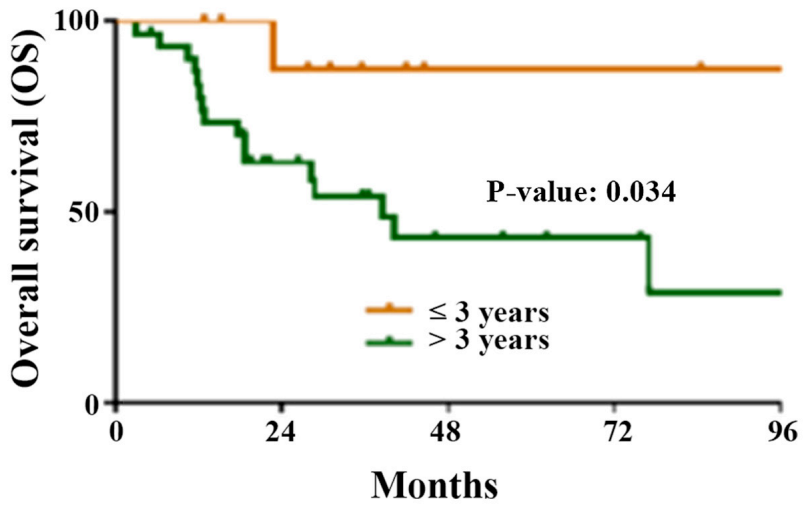

Figure 3 . OS in patients aged $<3$ years vs. patients aged $>3$ years $(\mathrm{P}=0.034)$. OS, overall survival.

was not reached ( $\mathrm{P}=0.034$; Fig. 3 ). Considering the 42 patients with HGGs, the therapeutic goal was to achieve, where possible, gross total resection following chemotherapy and radiotherapy, with as less structural damage as possible. A total of 11 patients underwent gross total resection (R0), while in patients receiving non-curative resection (R2), consisting of incomplete resection $(n=21)$ and biopsy $(n=10)$, the median OS were not reached (40.1 and 1.5 months, respectively; $\mathrm{P}=0.18)$.

Subsequently, $10 / 42$ patients $(23.8 \%)$ aged $<3$ years were administered HDCT with autologous bone marrow stem cell transplant. These patients did not reach median OS. Schedules with exclusive SDCT were administered to 22/42 patients (53.6\%), who demonstrated a median OS of 28.6 months, without a statistically significant trend ( $\mathrm{P}=0.08$; Fig. 4A). As regards SDCT, a temozolomide (TMZ)-based regimen was used in 17 cases and a platinum (CDDP)-based regimen in the remaining cases. The median OS of the patients who received CDDP-based treatment was 77 months, while it was 18.5 months for the patients who received TMZ-based treatment. No statistically significant differences in survival were observed between the TMZ- and CDDP-based chemotherapy regimens ( $\mathrm{P}=0.0849$; Fig. 4B). Regarding the palliative treatments, the progression-free survival (PFS) of the secondary and tertiary treatments was 6.5 and 2.1 months, respectively. The univariate analysis revealed a significant difference in the PFS curves between the primary (all patients or only patients with progressive disease) and subsequent treatments ( $\mathrm{P}=0.0001$, Fig. 5).

In addition, 24 patients (57.1\%) experienced disease progression, 14 of whom were subjected to a salvage second-observation surgery. The median OS of these patients was 28.7 months compared with 25.3 months when salvage surgery was not performed $(\mathrm{P}=0.957)$. Furthermore, 22 patients $(91.6 \%)$ received palliative multimodal anticancer treatments, including chemotherapy and radiotherapy, following disease progression.

\section{Discussion}

Pediatric HGGs are characterized by different molecular drivers, including mutations in the $\mathrm{H} 3$ histone variants $\mathrm{K} 27 \mathrm{M}$ (HIST1H3B and H3F3A), H3F3A G34R, BRAF V660E,
IDH and p53. The H3.3K27M mutation has been reported in several high-risk CNS malignancies, such as midline diffuse gliomas $(5,11,12)$. In particular, the K27M mutation is a well-established specific marker of pediatric midline glioma. The confirmation of the presence of this histone mutation is crucial for defining a new histological category, namely diffuse midline glioma with histone H3.3K27M mutation (10).

Previous studies have demonstrated that H3.3K27M drives a distinct subset of glial neoplasms of the CNS, depending not only on tumor type, but also on the site of origin and patient age. Gielen et al (2) analyzed 338 cases of pediatric brain tumors and revealed that the $\mathrm{H} 3.3 \mathrm{~K} 27 \mathrm{M}$ mutation was exclusively present in gliomas. In another study, the mutation rate was $80 \%$ in thalamic pediatric GBMs with a median age of 10.5 years (range, 5-23 years) $(13,14)$. Schwartzentruber et al reported the presence of the H3.3 variant in $31 \%$ of all pediatric GBMs; the $\mathrm{H} 3.3 \mathrm{~K} 27 \mathrm{M}$ mutation was more frequent compared with the $\mathrm{H} 3.3 \mathrm{G} 34 \mathrm{R} / \mathrm{V}$ mutation in pediatric diffuse high-grade astrocytomas, and the $\mathrm{H} 3.3 \mathrm{~K} 27 \mathrm{M}$ mutation primarily occurred in younger patients, whereas the H3.3G34R/V mutation was found in older patients and young adults (5).

Fontebasso et al suggested that every typical mutation in pediatric HGGs has a specific neuroanatomical position and a correlation with age; H3.3K27M occurs in children (1-10 years) and presents with a midline localization; and the IDH1 mutation occurs in adolescents (11-20 years), while the H3.3G34V/R occurs mutation in young adults (20-45 years), both of which occur in the cerebral hemispheres (15). Subsequent analysis demonstrated that the histone $\mathrm{H} 3.3 \mathrm{~K} 27 \mathrm{M}$ mutation was present in the majority of high-grade infiltrative astrocytomas arising within the midline structures (thalamus, brainstem and spinal cord) of pediatric and young adult patients. A high prevalence was particularly recorded in cases of thalamic GBM in young adults between 20 and 46 years of age, accounting for $90 \%$ (9/10 cases) of thalamic HGGs $(16,17)$.

In the present study, 24/42 patients were eligible for mutational analysis. The H3.3K27M mutation was identified in 7 patients $(29.1 \%)$. As regards tumor histology in patients with the H3.3K27M mutation, 3 were diagnosed with AAs and 4 with GBMs. No significant correlation was observed between tumor histology and the H3.3K27M mutation $(\mathrm{P}=0.6126)$. These findings were consistent with the findings of Karremann et al who also did not report any prognostic impact on the survival rates related to tumor histology (18).

It has been demonstrated that the presence of the H3.3K27M mutation in pediatric $\mathrm{HGG}$ was associated with an aggressive clinical behavior and a poor prognosis, including tumors that are histologically low-grade $(\mathrm{P}=0.025)(4,13,19)$. The multivariate analysis of the present study confirmed that H3.3K27M positivity was a significant prognostic factor $(\mathrm{P}=0.0045)$.

In a study published in 2018 involving 474 patients with pediatric $\mathrm{HGG}$, including 258 (54\%) patients with the H3K27M mutation and 216 (46\%) without the mutation, the presence of the mutation was independently and significantly associated with a worse prognosis $(\mathrm{HR}=3.630 ; \mathrm{P}<0.001)$. The patients with the mutation had a significantly shorter OS (by 2.3 years; $\mathrm{P}=0.008$ ) and they were $>3$ times more likely to succumb to the disease compared with $\mathrm{H} 3$ wild-type patients (20). However, a recent study concluded that the 
A

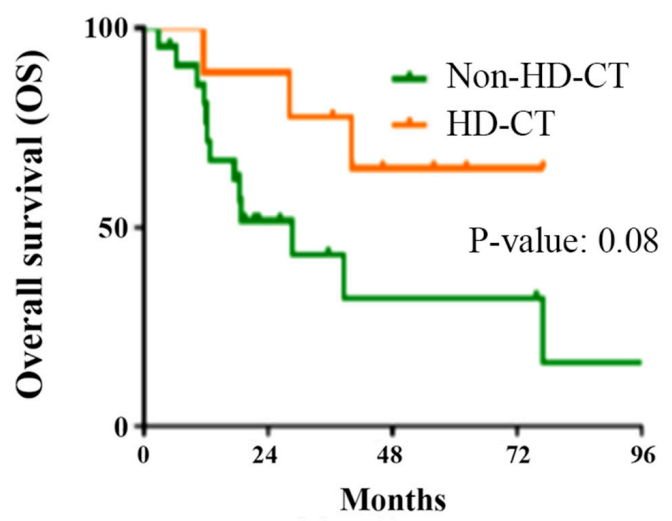

B

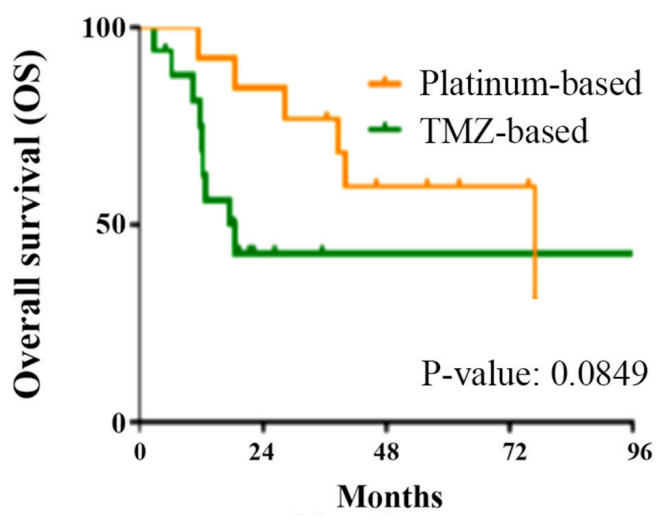

Figure 4. (A) OS in patients subjected to autologous bone marrow transplant treated with HDCT and patients treated with standard-dose chemotherapy (non-HD) $(\mathrm{P}=0.08)$. (B) OS in patients treated with TMZ-based and platinum-based regimens $(\mathrm{P}=0.0849)$. OS, overall survival; HDCT, high-dose chemotherapy; TMZ, temozolomide.

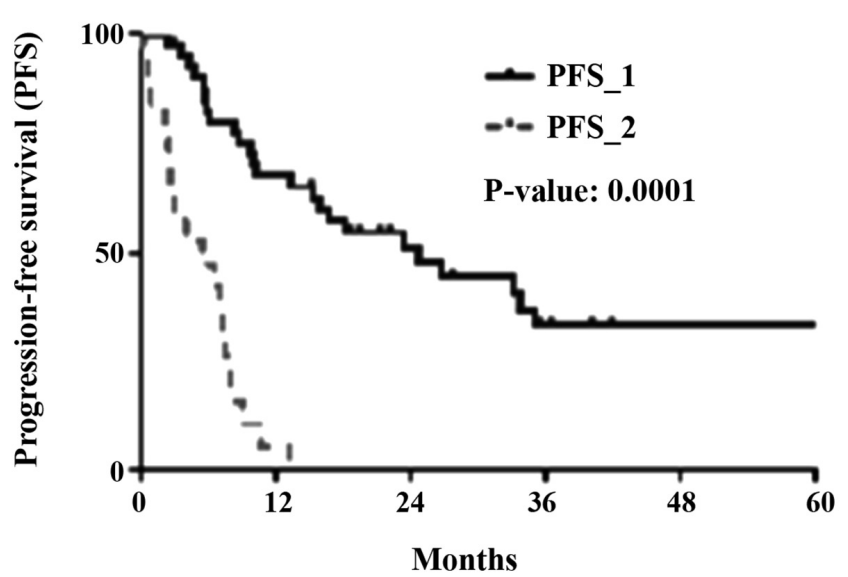

Figure 5. PFS of primary vs. secondary treatment (PFS-1 vs. PFS-2, respectively; $\mathrm{P}=0.0001)$. PFS, progression-free survival.

poor prognosis was due to the diffuse or infiltrative nature of the tumor; in fact, the prognosis of H3 wild-type diffuse gliomas remained significantly worse compared with that of the H3.3K27M-mutated gliomas (21).

The results of the present study indicated the important diagnostic and prognostic role of the H3.3K $27 \mathrm{M}$ mutation in pediatric gliomas. In addition, the role of the $\mathrm{H} 3.3 \mathrm{~K} 27 \mathrm{M}$ mutation may not be limited to the prognostic aspect, but it may also have therapeutic implications. Recently, retrospective studies analyzed the efficacy of histone deacetylase (HDAC) inhibitor drugs in patients with H3-mutant tumors. Felix and Fontenele (22) revealed a possible effect of valproic acid on the survival of patients with GBM through a HDAC-inhibiting effect. Thus, although this mechanism remains to be elucidated, $\mathrm{H} 3.3 \mathrm{~K} 27 \mathrm{M}$ mutations may make tumor cells more sensitive to HDAC inhibitors. Moreover, it has been hypothesized that the small molecule GSKJ4 may increase H3K27M methylation through inhibiting JMJD3 H3K27 histone demethylase; several in vitro studies have reported that patient-derived $\mathrm{H} 3.3 \mathrm{~K} 27 \mathrm{M}$ cell lines treated with GSKJ4 demonstrated decreased proliferation rates and cell viability (23). In addition, an ongoing phase 1 study at the University of California is currently evaluating the efficacy of a H3.3K27M-specific vaccine in HLA-A2 ${ }^{+}$children and young adults with H3.3K27M DIPGs and other HGGs. In that study, patients with high-risk glioma positive for HLA-A2 and the H3.3K27M mutation received the specific H3.3K27M peptide vaccine following radiotherapy (ClinicalTrials.gov Identifier: NCT02960230).

Interestingly, advanced studies have emphasized the discrepancies in H3.1 and H3.3 mutations, which have different clinical and prognostic characteristics. In the present analysis, only 1 patient with GBM carried a H1ST1H3B mutation, and the patient succumbed to the disease after the first round of therapy. Castel et al (8) reported that patients with H3.1 mutations exhibited an improved clinical response to radiotherapy with a more favorable OS compared with patients with H3.3 mutations. Thus, a more in-depth genetic characterization of the genetic signature of pediatric HGGs may help guide clinical trials with drugs that inhibit histone modifications. In addition to the effect of the treatment, it has been suggested that a more appropriate predictor of survival may be the type of histone $\mathrm{H} 3$ mutation rather than the clinical-radiological risk score. A multi-agent approach, inhibiting multiple targets, may increase treatment efficacy, exhibit synergy and possibly avoid the development of resistance (7).

The results obtained in the present study with regards to the presence of the BRAF V600E mutation, which, according to the literature, is associated with a poor prognosis, suggested its association with progressive disease with a poor response to conventional chemotherapy. It is therefore necessary to screen for BRAF V600E in LGGs, so that they may be re-evaluated over time, which may necessitate a different short-term and long-term therapeutic approach and eventually define early targeted therapies (9).

In conclusion, the results of the present study underlined the role of H3.3K27M and BRAF V600E mutations in pediatric gliomas, similar to previously reported studies. The present study validated the important diagnostic and prognostic role of these mutations in $\mathrm{HGG}$, highlighting the worse prognosis that accompanies H3K27M mutations and the high risk of progression in patients harboring BRAF V600E. Thus, the presence of histone mutations and all other mutations that characterize pediatric HGG is crucial and should be further investigated. 


\section{Acknowledgements}

The authors would like to thank Dr Maurizio Lucchesi for his valuable and constructive support during the development of this research. We also thank Dr Veronica De Gregorio for her technical assistance.

\section{Funding}

The present study was funded by 'La Forza di Giò-ovd' and Fondazione Anna Meyer- Firenze.

\section{Availability of data and materials}

All data generated or analyzed during the present study are included in this published article or are available from the corresponding author on reasonable request.

\section{Authors' contributions}

MG carried out literature search, study design, data collection, analysis and interpretation, figure preparation and manuscript writing. LGi carried out genetic analysis. AMB and CC carried out data collection and pathological analysis. MLC carried out statistical and data analysis. LGa and LGe carried out data collection and wrote the manuscript. IS conceved the study, analysed and interpreted data, and wrote the manuscript. All the authors have contributed to the critical revision of the manuscript. All the authors have read and approved the final version.

\section{Ethics approval and consent to participate}

The study protocol was approved by the Institutional Ethics Review Board and all patient data were derived from publicly available datasets.

\section{Patient consent for publication}

Not applicable.

\section{Competing interests}

The authors declare that they have no competing interests.

\section{References}

1. Merchant TE, Pollack IF and Loeffler JS: Brain tumors across the age spectrum: Biology, therapy, and late effects. Semin Radiat Oncol 20: 58-66, 2010.

2. Gielen GH, Gessi M, Hammes J, Kramm CM, Waha A and Pietsch T: H3F3A K27M mutation in pediatric CNS tumors: A marker for diffuse high-grade astrocytomas. Am J Clin Pathol 139: 345-349, 2013.

3. Bouffet E, Tabori U, Huang A and Bartels U: Possibilities of new therapeutic strategies in brain tumors. Cancer Treat Rev 36: $335-341,2010$.

4. Venneti S, Santi M, Felicella MM, Yarilin D, Phillips JJ, Sullivan LM, Martinez D, Perry A, Lewis PW, Thompson CB and Judkins AR: A sensitive and specific histopathologic prognostic marker for H3F3A K27M mutant pediatric glioblastomas. Acta Neuropathol 128: 743-753, 2014.

5. Schwartzentruber J, Korshunov A, Liu XY, Jones DT, Pfaff E, Jacob K, Sturm D, Fontebasso AM, Quang DA, Tönjes M, et al: Driver mutations in histone $\mathrm{H} 3.3$ and chromatin remodelling genes in paediatric glioblastoma. Nature 482: 226-231, 2012.
6. Bernstein BE, Mikkelsen TS, Xie X, Kamal M, Huebert DJ, Cuff J, Fry B, Meissner A, Wernig M, Plath K, et al: A bivalent chromatin structure marks key developmental genes in embryonic stem cells. Cell 125: 315-326, 2006.

7. Vanan MI, Underhill DA and Eisenstat DD: Targeting epigenetic pathway in the treatment of pediatric diffuse (high grade) gliomas. Neurotherapeutics 14: 274-283, 2017.

8. Castel D, Philippe C, Calmon R, Le Dret L, Truffaux N, Boddaert N, Pagès M, Taylor KR, Saulnier P, Lacroix L, et al: Histone H3F3A and HIST1H3B K27M mutations define two subgroups of diffuse intrinsic pontine gliomas with different prognosis and phenotypes. Acta Neuropathol 130: 815-827, 2015.

9. Lassaletta A, Zapotocky M, Mistry M, Ramaswamy V, Honnorat M, Krishnatry R, Guerreiro Stucklin A, Zhukova N, Arnoldo A, Ryall S, et al: Therapeutic and prognostic implications of BRAF V600E in pediatric low-grade gliomas. J Clin Oncol 35: 2934-2941, 2017.

10. Louis DN, Perry A, Reifenberger G, von Deimling A, Figarella-Branger D, Cavenee WK, Ohgaki H, Wiestler OD, Kleihues P and Ellison DW: The 2016 world health organization classification of tumors of the central nervous system: A summary. Acta Neuropathol 131: 803-820, 2016.

11. Rheinbay E, Louis DN, Bernstein BE and Suvà ML: A tell-tail sign of chromatin: Histone mutations drive pediatric glioblastoma. Cancer Cell 21: 329-331, 2012.

12. Wu G, Broniscer A, McEachron TA, Lu C, Paugh BS, Becksfort J, Qu C, Ding L, Huether R, Parker M, et al: Somatic histone H3 alterations in pediatric diffuse intrinsic pontine gliomas and non-brainstem glioblastomas. Nat Genet 44: 251-253, 2012.

13. Khuong-Quang DA, Buczkowicz P, Rakopoulos P, Liu XY, Fontebasso AM,BouffetE,Bartels U, AlbrechtS, Schwartzentruber J, Letourneau L, et al: K27M mutation in histone H3.3 defines clinically and biologically distinct subgroups of pediatric diffuse intrinsic pontine gliomas. Acta Neuropathol 124: 439-447, 2012.

14. Sturm D, Witt H, Hovestadt V, Khuong-Quang DA, Jones DT, Konermann C, Pfaff E, Tönjes M, Sill M, Bender S, et al: Hotspot mutations in H3F3A and IDH1 define distinct epigenetic and biological subgroups of glioblastoma. Cancer Cell 22: 425-437, 2012.

15. Fontebasso AM, Liu XY, Sturm D and Jabado N: Chromatin remodeling defects in pediatric and young adult glioblastoma: A tale of a variant histone 3 tail. Brain Pathol 23: 210-216, 2013.

16. Aihara K, Mukasa A, Gotoh K, Saito K, Nagae G, Tsuji S, Tatsuno K, Yamamoto S, Takayanagi S, Narita Y, et al: H3F3A K27M mutations in thalamic gliomas from young adult patients. Neuro Oncol 16: 140-146, 2014.

17. Buczkowicz P, Bartels U, Bouffet E, Becher O and Hawkins C: Histopathological spectrum of paediatric diffuse intrinsic pontine glioma: Diagnostic and therapeutic implications. Acta Neuropathol 128: 573-581, 2014.

18. Karremann M, Gielen GH, Hoffmann M, Wiese M, Colditz N, Warmuth-Metz M, Bison B, Claviez A, van Vuurden DG, von Bueren AO, et al: Diffuse high-grade gliomas with $\mathrm{H} 3$ K27M mutations carry a dismal prognosis independent of tumor location. Neuro Oncol 20: 123-131, 2018.

19. Korshunov A, Ryzhova M, Hovestadt V, Bender S, Sturm D, Capper D, Meyer J, Schrimpf D, Kool M, Northcott PA, et al: Integrated analysis of pediatric glioblastoma reveals a subset of biologically favorable tumors with associated molecular prognostic markers. Acta Neuropathol 129: 669-678, 2015.

20. Lu VM, Alvi MA, McDonald KL and Daniels DJ: Impact of the H3K27M mutation on survival in pediatric high-grade glioma: A systematic review and meta-analysis. J Neurosurg Pediatr 23: 308-316, 2018.

21. Pratt D, Natarajan SK, Banda A, Giannini C, Vats P, Koschmann C, Mody R, Chinnaiyan A and Venneti S: Circumscribed/non-diffuse histology confers a better prognosis in H3K27M-mutant gliomas. Acta Neuropathol 135: 299-301, 2018.

22. Felix $\mathrm{F}$ and Fontenele J: Valproic acid may be tested in patients With H3F3A-mutated high-grade gliomas. J Clin Oncol 34: 3104-3105, 2016.

23. Hashizume R, Andor N, Ihara Y, Lerner R, Gan H, Chen X, Fang D, Huang X, Tom MW, Ngo V, et al: Pharmacologic inhibition of histone demethylation as a therapy for pediatric brainstem glioma. Nat Med 20: 1394-1396, 2014. 